\title{
Estructura del capital: un estudio empírico de las principales aerolíneas colombianas
}

\author{
Judith Cecilia Vergara-Garavito* \\ Heyber Daney Durán-Parra** \\ Greyz Andrea Figueroa-López ${ }^{* * *}$
}

Fecha de recepción: 13 de febrero de 2020

Fecha de aceptación: 25 de junio de 2020

Resumen: La competitividad del mercado ha hecho replantear a las distintas aerolíneas su estructura de capital para lograr una combinación entre deuda y patrimonio que les permita obtener una mayor rentabilidad. Esta investigación tiene como objetivo identificar cuáles son los determinantes de la estructura de capital y qué teoría explica mejor las decisiones de financiación en las seis principales aerolíneas colombianas que transportan pasajeros. Para ello, se llevó a cabo una regresión de datos de panel, junto con un modelo de efectos fijos con la información semestral recopilada entre 2012 y 2017. Los resultados demuestran que la rentabilidad operacional, el crecimiento, la estructura de los activos y el tamaño de la empresa son los principales determinantes de la estructura de capital en las principales aerolíneas colombianas, alineándose esto con lo planteado por la teoría del equilibrio estático y la teoría de la jerarquía de preferencias.

Palabras clave: estructura de capital, industria aérea, rentabilidad, análisis económico, microeconomía.

Clasificación JEL: C33, D24, G32, G33, G34.

Cómo citar este artículo/ To reference this article / Comment citer cet article / Para citar este artigo:

Vergara-Garavito, J., Durán-Parra, H., \& Figueroa-López, G. (2020). Estructura del capital: un estudio empírico de las principales aerolíneas colombianas. Apuntes del Cenes, 39(70). Págs. 43 - 73

https://doi.org/10.19053/01203053.v39.n70.2020.10612

\footnotetext{
* Ph.D. en Administración Estratégica de Empresas. Profesora Departamento de Finanzas. Escuela de Economía y Finanzas, Universidad EAFIT. Medellín, Colombia. Correo electrónico:jvergar8@eafit.edu.co (D) https://orcid. org/0000-0002-7466-944X Autor para correspondencia.

** Magíster en Administración Financiera. Analista de Administración Deuda Corporativa, Avianca S.A. Bogotá D.C., Colombia. Correo electrónico: hdduranp@eafit.edu.co (iD https://orcid.org/0000-0002-6450-8132

*** Magíster en Administración Financiera. Contadora Pública. Universidad EAFIT. Bogotá D.C., Colombia. Correo electrónico:gafiguerol@eafit.edu.co (iD https://orcid.org/0000-0002-1066-8077
} 


\title{
Capital Structure: An Empirical Study of the Main Colombian Airlines
}

\begin{abstract}
The competitiveness of the market has led the different airlines to rethink their capital structure to achieve a combination of debt and equity, which allows them to obtain higher profitability. This research aims to identify the determinants of the capital structure and establish which theory explains in better way financing decisions in six Colombian airlines that transport passengers. To this end, a data panel regression was used with a fixed-effects model, considering semiannual information collected between 2012 and 2017. The results show that operational profitability, growth, asset structure and company size are the main determinants of the capital structure, aligning this with what is indicated in the trade off theory and the pecking order theory.
\end{abstract}

Keywords: capital structure; airline industry; profitability; economic analysis; microeconomics. 


\section{INTRODUCCIÓN}

El aumento en la demanda de transporte de pasajeros en las últimas décadas ha impulsado a las aerolíneas alrededor del mundo a buscar nuevas herramientas que les permitan ser más llamativas y competitivas para avanzar y no quedarse rezagadas en una industria que está en constante cambio. Según cifras del Banco Mundial (2019b), el número de pasajeros transportados por aerolíneas en el mundo ha venido aumentando de forma exponencial, pasando de 310 millones en 1970 a más de 3979 millones en 2017'. Este incremento se debió en parte al proceso de desregularización que sufrió el sector aéreo con la eliminación en 1978 de la Junta de Aeronáutica Civil (CAB) ${ }^{2}$ en los Estados Unidos, bajo la cual existían restricciones severas sobre la inversión y la operación de las aerolíneas que complicaban el panorama de crecimiento y desarrollo de la industria. Esta flexibilización de las políticas fue el gestor que permitió abrir un nuevo camino hacia la creación de aerolíneas o la fusión de existentes, reducir los precios de los pasajes, crear eficiencias operacionales y ampliar el servicio ofrecido a los pasajeros (Smith \& Cox, 2008).

Este proceso de desregularización desarrollado inicialmente en los Estados Unidos, poco a poco se fue ampliando a países de la región y de otros continentes, al ser considerado exitoso, toda vez que permitió la creación de decenas de nuevas aerolíneas que empezaron a competir en el precio, aprovechándose de una caída abrupta en los precios del petróleo, permitiéndoles reducir hasta en un $50 \%$ el precio de los pasajes, así como aumentar el número de rutas y frecuencias ofrecidas (Morris, 2014). Sin embargo, para algunos analistas y expertos de la industria como Brian Pearce (2017), economista jefe de la IATA, el proceso de desregularización no ha sido tan prometedor como se esperaba, pues 40 años después se han evidenciado problemas de monopolio $\mathrm{u}$ oligopolio, se ha creado una competencia agresiva entre las aerolíneas que ha

1 Estadísticas mundiales de aviación civil y estimaciones de personal de la Organización de Aviación Civil Internacional (oaci).

2 En inglés, Civil Aeronautics Board. 
generado que varias terminen acogiéndose a leyes de quiebra, han aumentado los costos y reducido la rentabilidad debido a la volatilidad en los precios del petróleo y de las tasas de cambio y, principalmente, se han dañado los modelos de servicio al cliente (Leggett, 2011).

El sector aéreo colombiano no ha sido ajeno a este proceso. Desde la última década, la industria de la aviación ha experimentado cambios importantes debido al desarrollo tecnológico que ha traído el proceso de globalización al país, en cuanto a infraestructura y a renovación de la flota en las distintas aerolíneas, así como al mejoramiento de las políticas públicas, al punto de convertirlo en un servicio público esencial para la economía (Díaz, 2015). A pesar de las dificultades del entorno económico mundial, según la Autoridad de Aviación Civil (AAC), desde 2005 el transporte de pasajeros por vía aérea ha venido en aumento, llegando a 37,78 millones de personas movilizadas en 2018, un aumento del $6.1 \%$ respecto a 2017, de los cuales cerca de 24 millones de pasajeros se movilizaron al interior del país y 13 millones 756000 a destinos internacionales, un incremento del $12.1 \%$ respecto al año anterior, posicionando al mercado colombiano en el tercer lugar después de Brasil y México en América Latina (Aeronáutica Civil de Colombia, 2017).

El 2018 fue, sin duda, el año con la mayor movilización de pasajeros de la historia, debido principalmente al aumento del sector turismo, la inversión privada y el mejoramiento de la infraestructura aeroportuaria, así como a la autorización de nuevas rutas y el aumento de frecuencias (Aeronáutica Civil, 2019b).

Sumado a lo anterior, la volatilidad y el dinamismo del sector generan una necesidad constante en las empresas de optimizar su estructura de capital con el fin de cumplir con sus requerimientos de financiación e inversión. Estrategias que deben implementarse sin dejar de lado proyectos que le permitan atender la creciente demanda de pasajeros y la búsqueda de elementos diferenciadores para ofrecer un servicio llamativo y de calidad. Lo anterior debe estar alineado con el entorno macroeconómico del país y del mundo, puesto que es un factor importante a la hora de determinar el nivel de endeudamiento que las compañías pueden afrontar, teniendo en cuenta la alta dependencia de fuentes externas de financiación.

Es así como el presente documento identifica algunos de los determinantes de financiación de capital para las seis principales compañías de transporte aéreo de pasajeros en Colombia, contemplando las necesidades de inversión en capital dirigidas a la generación de rentabilidad, adquisición de aeronaves, optimización de los costos variables y minimización de los gastos de ventas y operación. Todo esto sin dejar atrás variables de exposición al riesgo de mer- 
cado que tiene el sector, especialmente las referentes a tasa de interés, precios del combustible y tipo de cambio.

Con base en lo anterior, el presente estudio plantea la posibilidad de contrastar empíricamente para el caso de las aerolíneas colombianas, cuál de las teorías propuestas en la literatura es la que mejor explica la estructura de capital que usan las firmas, y así establecer si existe una adecuada estructura de capital de acuerdo con una serie de variables que influyen en el nivel de apalancamiento de las compañías analizadas. El artículo se organiza de la siguiente manera: primero, la sección que corresponde al marco teórico. Seguidamente se presenta la sección referente a la metodología, así como la definición de las variables y del modelo utilizado. La siguiente sección corresponde a los resultados y finalmente se presentan las conclusiones.

\section{MARCO TEÓRICO}

A mitad del siglo xx empezaron a surgir teorías que intentaban explicar la estructura de capital de las empresas, con el ánimo de determinar cuál era la composición óptima que deberían tener las firmas para incrementar su valor $y$ alcanzar un costo de capital mínimo. A partir de este enfoque y bajo un supuesto de mercados perfecto, aparece la tesis tradicional con David Durand en 1952 y la tesis de irrelevancia de Modigliani y Miller (M\&M) en 1958, quienes propusieron una reformulación de la tesis tradicional (Rivera, 2002). Posteriormente, bajo la hipótesis de mercados imperfec- tos se desarrollan las principales teorías modernas, como la teoría del equilibrio estático o trade-off de Myers (1977), la teoría de los costos de agencia de Jensen y Meckling (1976) y la teoría de orden jerárquico o selección de preferencias (Pecking Order) de Myers (1984).

Bajo la tesis tradicional se encuentran los aportes entregados por autores como Graham y Dodd (1940) y Durand (1952) como su principal exponente, así como los de Guthmann y Dougall (1955) y Schwartz (1959). Dentro de este enfoque se busca la estructura de capital óptima, haciendo uso del apalancamiento financiero debido al bajo costo en que se incurre frente al que exigen los inversionistas de capital, lográndose así un aumento en el valor de la empresa. No obstante, el apalancamiento debe usarse de forma moderada, puesto que al aumentar el mismo los accionistas exigirán una mayor tasa de retorno hasta el punto en que las exigencias de los inversionistas compensen el uso de la deuda, aumentado el costo de capital y disminuyendo el valor de la empresa (Zambrano \& Acuña, 2011).

En su trabajo, Durand (1952) asegura que el uso moderado de la deuda incrementará el valor de la compañía, siempre y cuando las exigencias de los accionistas sean menores al beneficio de incrementar el apalancamiento. De ahí que la combinación de deuda y capital que logre una estructura de capital óptima debe ser definida por los directivos de la compañía. En su estudio también 
analiza que no siempre la maximización de las utilidades genera un mayor valor de la compañía, por lo que el directivo u hombre de negocios debe centrarse en la maximización del valor presente de sus flujos futuros y no en la maximización de la utilidad actual.

Por otro lado, Modigliani y Miller (1958) propusieron que el valor de las empresas no se ve afectado por la estructura de capital bajo el supuesto de mercados perfectos, es decir, no existen imperfecciones de mercado tales como los impuestos, costes de transacción, asimetría de información, entre otros, haciendo irrelevante las decisiones de estructura financiera. Su postulado estaba basado en dos proposiciones: el valor de mercado de la empresa y el costo de capital. La primera expone que el valor de mercado de una empresa y el costo de capital son independientes de su estructura de capital, es decir, que el valor de la empresa no depende de su financiación; la segunda indica que el valor de la empresa está dado por el potencial que tiene para generar ganancias y del riesgo de los activos que posee sin determinar la forma en que son financiados los activos. Esta última proposición se podría expresar de la siguiente forma:

$$
K_{e}=k_{0}+\frac{D}{E} \times\left(k_{0}-k_{d}\right)
$$

Donde:

$K_{e}=$ rendimiento esperado o costo del capital $k_{0}=$ costo del capital o waCC sin apalancamiento

$k_{d}=$ costo de la deuda

$\frac{D}{E}=$ ratio deuda-equity de la empresa

Dicho análisis demostró que el valor de la empresa está determinado por sus resultados operativos; además se evidencia una independencia entre el costo promedio del capital y el valor de la empresa, lo que lleva a inferir que no hay una estructura de capital óptima. No obstante, sus críticos indicaban que esta teoría no era aplicable debido a la ausencia de las imperfecciones del mercado, tales como los beneficios en impuestos que favorecen la adquisición de deuda, lo que llevó a Modigliani y Miller a replantear algunos de sus postulados en 1963, con la publicación de correcciones a su artículo (Azofra \& Fernández, 1999).

\section{Teoría del equilibrio estático o Trade Off}

A partir de la propuesta de Modigliani y Miller (1958), aparece la teoría del Trade Off, la cual sostiene, bajo el supuesto de mercados imperfectos, que las compañías buscan una relación deuda/capital óptima que maximice su valor, de acuerdo con un nivel de apalancamiento objetivo que permita un equilibrio entre los beneficios y costos marginales producto del endeudamiento (Myers, 1977). Esta teoría argumenta que las empresas alcanzarán un nivel de endeudamiento hasta que el valor marginal del beneficio en impuestos, 
producto de la deuda, se disminuya por el valor actual de los costos de quiebra o reorganización (Shyam \& Myers, 1999).

A diferencia de la teoría de Modigliani y Miller, en donde se argumentaba que las empresas deben endeudarse tanto como puedan, la teoría del Trade Off sugiere que las compañías deben manejar niveles de endeudamiento moderados para no sufrir dificultades financieras derivadas del atractivo que puede generar endeudarse para financiarse por las ventajas impositivas que trae, lo que a largo plazo podría materializarse en un riesgo financiero y en una pérdida de valor de mercado de la empresa.

\section{Teoría de costos de agencia}

Esta teoría propuesta por Jensen y Meckling (1976) analiza los conflictos que se pueden generar en las organizaciones entre sus diferentes grupos de interés, los cuales se conocen como costos de agencia. Los conflictos se dan entre el principal (dueños de la empresa) y el agente (directivos, gerentes) debido a que cada uno busca maximizar su beneficio; el agente en cuanto al control sobre la empresa y el principal en cuanto al incremento del valor de esta (Ross, 1977). La teoría plantea el uso de la deuda como una herramienta que tienen los accionistas para controlar las decisiones de la administración, dado un nivel de apalancamiento, que logra un mayor control sobre las estrategias del agente, lo que lleva a reducir los costos de agen- cia y maximiza el valor de la empresa, obteniendo una estructura de capital óptima (Zambrano \& Acuña, 2011).

\section{Teoría de las jerarquías de preferen- cias o Pecking Order}

Durante el mismo periodo en el que se desarrolla la teoría del Trade Off, se empezaba a construir la teoría de la jerarquización financiera o Pecking Order (Myers, 1984), la cual afirma que la estructura de capital óptima no existe, sino que, por el contrario, las empresas eligen sus fuentes de financiación en función de los costes de cada uno, recurriendo primero al financiamiento interno, que tiene un menor costo, luego a emisión de deuda y finalmente a emisión de acciones (Murray \& Vidhan, 2005).

La teoría se analiza bajo un entorno de asimetría de información entre las empresas y el mercado de capitales, debido a que las empresas tienen más información sobre su estado actual, a diferencia de los inversionistas, quienes solo poseen la información que les comparten y una aproximación resultante de análisis comparativos y estimativos (Zambrano \& Acuña, 2011). Esta teoría es ampliamente reconocida en la actualidad, debido a que las compañías no están tras una relación deuda/capital óptima, sino que buscan que sus proyectos de inversión se puedan realizar con la reinversión de utilidades a un menor costo (Myers, 1984). 
Existen varios estudios empíricos en el mundo sobre estructura de capital en empresas del sector aeronáutico, en los que se analizan las principales variables determinantes de la estructura de capital. Es el caso de la investigación de Kasim y Nurhan (2018), quienes ponen a prueba los factores que determinan la estructura de capital, planteados en las diferentes teorías clásicas y modernas, en un conjunto de 15 compañías aéreas de bajo costo o Low Cost alrededor del mundo entre 2004 y 2015, con el fin de revelar si su estructura de capital responde a alguna de las teorías existentes. Además, buscan brindar herramientas a gerentes y directivos de estas compañías para la toma de decisiones de inversión y financiación. En cuanto a los resultados obtenidos, las principales variables que se destacan son el tamaño de la empresa, que tiene una relación positiva con el nivel de apalancamiento, el crecimiento, la tangibilidad de los activos y el escudo fiscal y, así mismo, comprueban que las variables responden a los postulados de las teorías modernas.

Por otro lado, Sewunet (2017) presenta los determinantes internos y externos de la estructura de capital en la industria aérea, enfocándose en tres de las principales aerolíneas del África Subsahariana para el periodo 2002-2015, con el fin de analizar cómo están influenciadas las decisiones de financiación de las empresas aéreas en el continente africano para que estas aumenten su valor, así como corroborar la aplicación de las diferentes teorías para explicar la estructura de capital. Por medio de un modelo de efectos fijos encontraron que los activos, la inflación $\mathrm{y}$ el riesgo tienen una relación positiva con el apalancamiento, mientras que la rentabilidad y el tamaño tienen una relación negativa.

Por otra parte, Alahyari (2014) hizo un estudio sobre los determinantes de la rentabilidad en la aerolínea turca Turkish Airlines y encontró que el tamaño de la empresa se relaciona con su tasa de rentabilidad, pues a medida que aumenta el tamaño, se generan economías de escala y maximización del valor de esta. En cuanto al crecimiento y su relación con la rentabilidad, se evidenció que con alto crecimiento se tienen mejores expectativas sobre el futuro de la empresa, lo que genera un mejor rendimiento y una mayor rentabilidad. Los resultados del estudio arrojaron que las oportunidades de crecimiento, el valor de garantía de los activos y el índice de liquidez son los principales determinantes de la rentabilidad.

Uno de los determinantes más importantes de la estructura de capital en la industria aérea es el arrendamiento o leasing, lo que llevó a Erickson y Trevino (1994) a realizar una investigación sobre los determinantes del arrendamiento, tanto a corto como a largo plazo, en empresas de la industria aérea entre 1985 y 1990, para establecer si los arrendamientos son sustitutos o complementos de la deuda. Así pues, a pesar de no existir modelos ampliamen- 
te aceptados sobre los determinantes del arrendamiento, lograron establecer que existe una serie de factores para tener en cuenta, como el factor impositivo del arrendamiento, siendo este el principal, debido a la naturaleza de las operaciones realizadas por las aerolíneas, las cuales no cuentan en su mayoría con flota propia sino arrendada, situación que genera beneficios fiscales al trasladarle la carga impositiva a los arrendadores.

Los resultados demuestran que la rentabilidad y el crecimiento son determinantes potencialmente importantes del arrendamiento y que los arrendamientos financieros son un sustituto de la deuda, mientras que los arrendamientos operativos no lo son. También se demostró que al controlar la rentabilidad y el crecimiento, los ratios de arrendamiento se relacionan inversamente con los ratios de deuda.

Entre tanto, Akgül y Sigali (2018) analizaron los determinantes de las decisiones de estructura de capital en aerolíneas turcas que han cotizado en la bolsa de Estambul entre 2002 y 2013, pues consideraban que, dada su posición geográfica, Turquía era un importante centro de operaciones en la región, motivo por el cual se debían analizar las principales variables que determinan las decisiones de financiación de las empresas. Los resultados demuestran que la rentabilidad no es un determinante de la estructura de capital, mientras que el tamaño y los activos sí lo son. Lo cual sugiere que en una economía emergente como la turca, las empresas de mayor tamaño tienen mayor credibilidad y acceso a más fuentes de financiación.

Diferentes estudios sobre estructura de capital también buscan determinar la relación que existe entre esta y el valor de la empresa. Es así como Tomoiagă (2014) busca establecer que de acuerdo con la estructura de capital adoptada por 111 empresas aéreas alrededor del mundo que cotizaban en la bolsa en 2014, existe un impacto en su valor de mercado. Los resultados arrojan que la mayoría de las empresas tienen una estructura de capital similar y que el tamaño y los activos son determinantes entre la relación estructura de capitalvalor de la empresa, en donde el tamaño tiene una relación negativa y los activos tienen una relación positiva.

En esa misma línea, Capobianco y Fernandes (2004) presentan un artículo en donde ponen a prueba la hipótesis de que, dado un nivel de apalancamiento razonable, las empresas tendrán un rendimiento financiero eficiente con un nivel bajo de activos. Para tal fin, recopilaron información entre 1993 y 1997 de las mayores compañías aéreas del mundo en donde el capital de los accionistas representaba menos del $40 \%$ del capital total. Los resultados arrojaron que existe una relación directa con el nivel de eficiencia para aquellas empresas que tienen bajo endeudamiento y alto rendimiento, mientras que no se encontró una relación para empresas con bajo rendimiento y alto endeudamiento. 
Finalmente, es importante resaltar el estudio de Khalil (2017), pues es un referente para la presente investigación, debido a que el autor analiza los principales determinantes de la estructura de capital de ocho grandes aerolíneas, con factores diferenciadores, entre ellos el nivel de costo, servicio al cliente y posición financiera, para un periodo de cinco años y cuyo objetivo es identificar cuáles de las 13 principales variables sobre estructura de capital, que se recopilaron de la revisión de literatura, son aplicables a estas compañías. En él se argumenta cuán importante es la elección de una adecuada estructura de capital, pues es una decisión estratégica de la gerencia por las implicaciones de costos y riesgo asociados que puede traer a las compañías frente al beneficio generado. Estas decisiones se deben tomar teniendo en cuenta la particularidad de cada empresa y no generalizando un solo modelo para todas ellas. Bajo un análisis de regresión de datos de panel se encontró que las ganancias, el tamaño de la compañía, el arrendamiento, los activos y la diferencia entre retorno y retorno promedio son los determinantes de la estructura de capital en las aerolíneas estudiadas.

\section{METODOLOGÍA}

Para la presente investigación se consultó la clasificación de las aerolíneas en Colombia por actividad, entregada por la Aerocivil, la cual se presenta en la Figura 1.

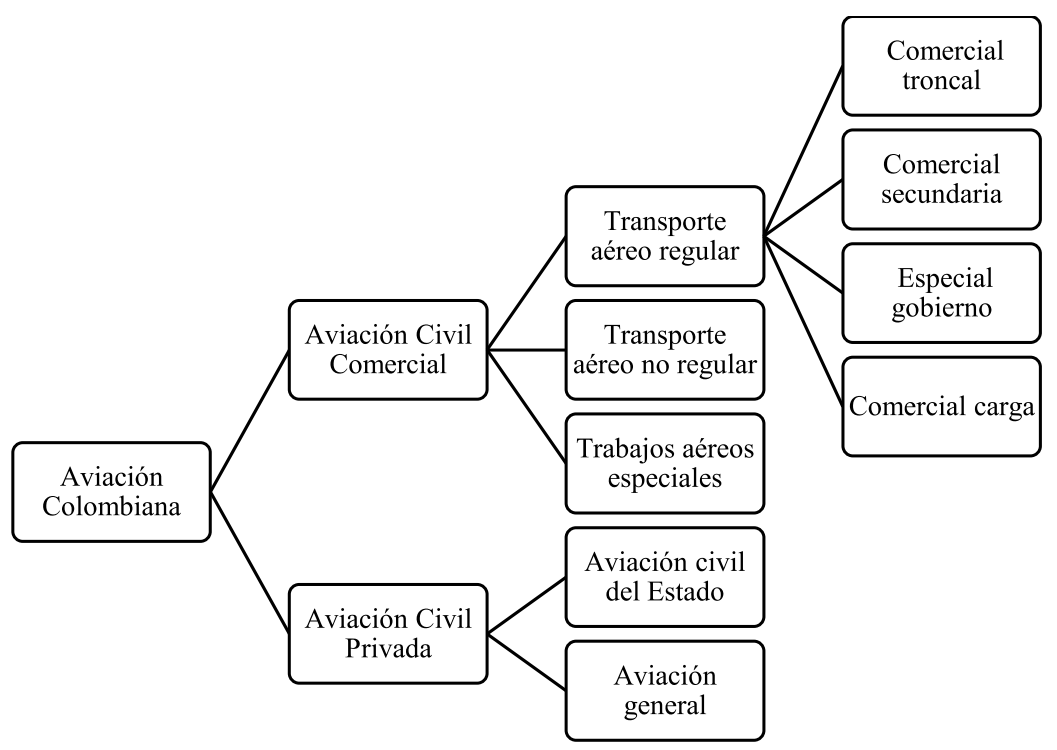

Figura 1. Clasificación de la aviación colombiana 
Teniendo en cuenta el alcance de esta investigación, se seleccionaron seis aerolíneas de transporte de pasajeros de acuerdo con su modalidad de transporte: Avianca (2017, 2018), LATAm (2017, 2019), Copa Airlines (2017), Viva Air (2018), Easyfly (2019) y Satena (2019). La información sobre estas empresas se recopiló para los periodos semestrales comprendidos entre 2012 y 2017, y se obtuvieron los datos de fuentes secundarias, como los estados financieros e informes de gestión publicados en las páginas oficiales, reportes entregados por entes de vigilancia y control, artículos, investigaciones, entre otros, con una técnica de recolección mixta (cuantitativo-cualitativo) de la información. Los datos fueron analizados con el software estadístico E-Views 11.

Para la presente investigación se llevó a cabo una estimación mediante la metodología de datos de panel a seis aerolíneas colombianas, para los periodos semestrales comprendidos entre 2012 y 2017, con el fin de validar si las variables explicativas propuestas determinan el nivel de apalancamiento de las empresas. Se recurre al método de regresión de datos de panel, debido a que permite combinar dos dimensiones: tiempo y agentes económicos, también denominadas dimensión temporal y estructural. Utilizar esta técnica tiene una serie de ventajas en comparación con los modelos de series de tiempo y de corte transversal (Baronio \& Vianco, 2014):
- Permite al investigador ampliar el número de observaciones $(\mathrm{n} \times \mathrm{T})$, mejorando la eficiencia de las estimaciones.

- Permite capturar la heterogeneidad no observable entre agentes como en el tiempo.

- Supone e incorpora en el análisis que los individuos son heterogéneos.

- Permite que potencialmente los problemas de multicolinealidad puedan reducirse.

Como la estimación por el método de mínimos cuadrados ordinarios (MCO) podría estar sesgada a causa de la existencia de errores correlacionados, existen dos procedimientos adicionales para evitar el problema de sesgo sobre los estimadores de los parámetros de las variables explicativas: el método de efectos fijos y el método de efectos variables. Para esta investigación se utilizará el método de efectos fijos, pues reconoce que las variables omitidas podrían generar cambios en los interceptos, ya sea por medio del tiempo o por unidades de corte transversal, por lo que transforma el modelo incluyendo variables dummy que recojan la característica diferencial de cada individuo (Baronio \& Vianco, 2014).

Definición de variables y modelo empírico

De acuerdo con la revisión de la literatura, la presente investigación reúne una serie de variables que pueden ser 
aplicables en las empresas objeto de estudio. Como variable dependiente está el nivel de endeudamiento y como independientes están rentabilidad, estructura de los activos, crecimiento, combustible, arrendamiento, tamaño de la compañía, riesgo de la empresa y liquidez. Así mismo, fueron consideradas variables dummy como estrategia de negocio, si la empresa es pública o privada, ciclo económico y si la empresa pertenece a un holding.

\section{Variable dependiente}

La variable dependiente se podrá calcular de dos formas: según el nivel de apalancamiento o la ratio de apalancamiento.

\section{Nivel de apalancamiento}

El nivel de apalancamiento refleja el porcentaje de participación de los acreedores dentro de la empresa (Ortiz, 2011).

$$
\mathrm{NA}=\frac{\mathrm{TPC}}{\mathrm{TA}}
$$

$\mathrm{NA}=$ Nivel de apalancamiento $\mathrm{TPC}=$ Total pasivo con terceros $\mathrm{TA}=$ Total activo

\section{Ratio de apalancamiento}

La ratio de apalancamiento representa el porcentaje de deuda en relación con los fondos propios de la empresa $\mathrm{y}$ observa en qué cuantía la financiación afecta el equilibrio y desarrollo de la misma (Pedra, 2016).

$$
\mathrm{RA}=\frac{\mathrm{PC}+\mathrm{PNC}}{\mathrm{TA}}
$$

$\mathrm{RA}=$ Ratio de apalancamiento

$\mathrm{PC}=$ Pasivo corriente

PNC $=$ Pasivo no corriente

$\mathrm{PT}=$ Patrimonio neto

\section{Variables independientes}

Las variables independientes utilizadas en la construcción del modelo se presentan y definen a continuación.

\section{Rentabilidad operacional}

La variable de rentabilidad operacional refleja el beneficio y crecimiento esperado de la empresa, en relación con sus ingresos operacionales (Khalil, 2017). El presente estudio define como base para cálculo de la variable, la utilidad operacional reportada por la Aeronáutica Civil en sus estadísticas anuales.

$$
\mathrm{RO}=\frac{\mathrm{UO} t}{\mathrm{IT} t}
$$




\section{Estructura de los activos}

La estructura de activos refleja la disponibilidad de activos de la empresa, puede tener relación con el nivel de apalancamiento y la generación de valor en la compañía (Kasim \& Nurhan, 2018). Para el presente estudio, los activos fijos se presentan en valor neto, es decir, una vez descontada la depreciación acumulada.

$$
\mathrm{EA}=\frac{\mathrm{AF} t}{\mathrm{TA} t}
$$

$\mathrm{EA}=$ Estructura de activos

$\mathrm{AF}=$ Activos fijos

$\mathrm{TA}=$ Total activos

\section{Crecimiento}

Esta variable mide la variación porcentual de los ingresos de la compañía de un año respecto a otro. El crecimiento se ve afectado por factores como el retorno del negocio, niveles de demanda, competencia empresarial, entre otros. Al mismo tiempo guarda relación con el nivel de apalancamiento de la empresa e impacta en la rentabilidad del negocio (Khalil, 2017).

$$
\mathrm{C}=\left(\left\{\frac{\operatorname{IT}(t)}{\operatorname{IT}(t-1)}\right\}-1\right)
$$

$\mathrm{C}=$ Crecimiento

IT $=$ Ingresos totales

\section{Combustible}

El combustible es uno de los factores más importantes y representativos en el esquema de costos y gastos de las aerolíneas, al impactar directamente la rentabilidad y el nivel de apalancamiento del negocio. Esta variable se encuentra directamente relacionada con el nivel de ventas de las aerolíneas y su fluctuación depende de factores macroeconómicos, geopolíticos, entre otros (Briceño \& Lopez, 2011).

Entre los estudios empíricos revisados se encontraron trabajos con aspectos relacionados con la estructura de costos en las aerolíneas, como el desarrollado por la Oficina de Regulación Económica del Ministerio de Transporte de Colombia en 2009, donde se muestra la participación del costo por combustible de algunas aerolíneas colombianas (Oficina de Regulación Económica-Ministerio de Transporte, 2009).

Considerando la limitación de información financiera de las diferentes aerolíneas, se han tomado como base para el cálculo de la variable, las estadísticas de las actividades aeronáuticas publicadas por la Aeronáutica Civil, específicamente el costo promedio de gasto por combustible de las aerolíneas para cada semestre entre los años 2012 a 2017 (Aeronáutica Civil de Colombia, 2019a). Para el caso de Satena y Avianca, el gasto por combustible ha sido sustraído 
directamente de las notas a los estados financieros separados de las compañías para los periodos 2012 a 2017.

$$
\mathrm{C}=\frac{\mathrm{GC}}{\mathrm{CGT}}
$$

$\mathrm{C}=$ Combustible

$\mathrm{GC}=$ Gasto en combustible

$\mathrm{CGT}=$ Costos y gastos totales

\section{Arrendamiento}

Esta variable indica la participación del gasto por arrendamiento operativo en los ingresos de la compañía, con el fin de determinar su influencia en el apalancamiento y rentabilidad del negocio. Desde el punto de vista de estructura de capital, (Damodaran, 2019) expresa el gasto de arrendamiento operativo (Lease Expense/Sales) como el 5.34 \% de las ventas totales, porcentaje calculado a enero de 2019 sobre la información financiera de 162 empresas pertenecientes a la industria aérea global, donde se incluyen regiones como Estados Unidos, Europa, Japón, Australia, Nueva Zelanda, Canadá y algunos mercados emergentes.

Como referencia para el cálculo de la variable se han tomado las estadísticas de las actividades aeronáuticas publicadas anualmente por la Aeronáutica Civil, específicamente el costo promedio de gasto por arrendamiento de las aerolíneas para cada semestre entre los años 2012 a 2017 (Aeronáutica Civil de Colombia, 2019a). Para el caso de Satena y Avianca, el gasto por arrendamiento ha sido tomado directamente de las notas a los estados financieros separados de las compañías, para los periodos 2012 a 2017.

$$
\mathrm{A}=\frac{\mathrm{GAO}}{\mathrm{CGT}}
$$

$\mathrm{A}=$ Arrendamiento

$\mathrm{GAO}=$ Gasto en arrendamiento operativo

CGT $=$ Costos $\mathrm{y}$ gastos totales

\section{Tamaño de la compañía}

$$
\mathrm{T}=\operatorname{Ln}(\mathrm{IT})
$$

$\mathrm{T}=$ Tamaño

IT $=$ Ingresos totales

Mediante esta variable se busca conocer la relación que existe entre el tamaño y el nivel de endeudamiento de la compañía. En su estudio, Khalil (2017) ilustra el tamaño de la compañía como el Ln de las ventas, dada la influencia en los ingresos de la empresa, el impacto directo en la rentabilidad del negocio y la relación positiva que existe entre el tamaño y el apalancamiento de la empresa, según la teoría del Trade Off. De igual manera, Titman y Wessels (1988), relacionan la variable tamaño con el nivel de apalancamiento de la compañía argumentando una diferenciación de las grandes empresas respecto a las pequeñas en términos de diversificación 
del riesgo y necesidades de financiación. Para la presente investigación se tomará como referencia la ecuación antes mencionada.

\section{Riesgo de la empresa}

El riesgo de la empresa está explicado como la desviación estándar de su utilidad operacional y es utilizado por las empresas para expresar la probabilidad de tener dificultades financieras o declararse en quiebra (Kasim \& Nurhan, 2018). Según lo desarrollado en la teoría del Trade Off, las empresas tienen más probabilidad de tener problemas financieros o de quiebra cuando sus flujos de efectivo futuros son inciertos, lo que podría llegar a generar un impacto negativo en su capacidad de endeudamiento y aumentar la posibilidad de incumplimiento de sus obligaciones financieras. Por lo tanto, una empresa con alto riesgo tiene más probabilidades de tener un nivel bajo de endeudamiento (Demirhan, 2009).

$$
\mathrm{R}=\mathrm{DEUO}
$$

$\mathrm{R}=$ Riesgo

DEUO = Desviación estándar de la Utilidad Operacional

\section{Liquidez}

Mediante el índice de liquidez se busca medir la relación que existe con el nivel de endeudamiento de la empresa, dada una estructura de derechos y obligaciones a corto plazo.

$$
\mathrm{L}=\frac{\mathrm{AC}}{\mathrm{PC}}
$$

$$
\begin{aligned}
& \mathrm{AC}=\text { Activo corriente } \\
& \mathrm{PC}=\text { Pasivo corriente }
\end{aligned}
$$

\section{Estrategia de negocio}

Las aerolíneas Low Cost o de bajo costo son aquellas que ofrecen tarifas bajas en detrimento de otros servicios ofrecidos a los usuarios. Esta estrategia permite una reducción significativa de la estructura de costos y una mayor eficiencia en el uso de los recursos (Barcelonactiva, 2012). Se utilizará una variable binaria para captar el efecto que pueda tener este enfoque de negocio. Tomará el valor 1 si tiene estrategia de bajo costo y 0 en caso contrario.

\section{Privado o público}

Se incluye esta variable binaria para medir el impacto que puede tener sobre la estructura de capital la naturaleza de la empresa, si es pública o privada, pues existen diferencias en las visiones de negocio que cada una persigue, así como en el respaldo financiero que pue- 
den ofrecer. Tomará el valor de 1 si la participación del estado en la empresa supera el $50 \%$ y cero en caso contrario.

\section{Ciclo económico}

$\mathrm{Al}$ incluir esta variable se pretende captar el efecto que pueden tener los ciclos económicos de contracción o recesión sobre las decisiones de financiación de las aerolíneas, debido a la sensibilidad de la industria a cambios en la economía. La economía colombiana ha tenido momentos difíciles, como en 2012 con la crisis de Interbolsa, cuando el sistema de inversión en el mercado de capitales se vio afectado ${ }^{3}$, y en 2015 con la caída de los precios internacionales del petróleo (Romero, 2019). Así lo demuestra el Banco Mundial en su reporte de cifras históricas del crecimiento del PIB anual hasta 2017 para Colombia (Banco
Mundial, 2019a). Tomará el valor de 1 si se presentó una contracción en el ciclo económico o 0 en caso contrario.

\section{Pertenece a un holding}

Pertenecer a un holding puede traer beneficios adicionales al momento de adquirir nueva deuda, puesto que la empresa puede acceder a créditos de mayor valor con tasas más bajas por contar con el respaldo de una estructura empresarial (International Credit Rating, 2018). La variable tomará el valor de 1 si la empresa pertenece a un holding o 0 en caso contrario.

\section{Modelo empírico}

De acuerdo con las variables anteriormente definidas, el modelo de regresión de datos panel propuesto para la presente investigación es el siguiente:

$$
\begin{aligned}
Y_{t i}= & \beta_{0}+\beta_{X 1} X 1_{i t}+\beta_{X 2} X 2_{i t}+\beta_{X 3} X 3_{i t}+\beta_{X 4} X 4_{i t}+\beta_{X 5} X 5_{i t}+\beta_{X 6} X 6_{i t} \\
& +\beta_{X 7} X 7_{i t}+\beta_{X 8} X 8_{i t}+\beta_{d 1} d 1_{i t}+\beta_{d 2} d 2_{i t}+\beta_{d 3} d 3_{i t}+\beta_{d 4} d 4_{i t}+u_{i t}
\end{aligned}
$$

Donde:

$Y_{t i}=$ nivel de apalancamiento

$\beta_{0}=$ intercepto

$X 1_{i t}=$ rentabilidad operacional de la empresa i en el momento t.

$X 2_{i t}=$ estructura de activos de la empresa i en el momento $t$.

$X 3_{i t}=$ crecimiento de la empresa i en el momento $t$.

$X 4_{i t}=$ costo del combustible de la empresa i en el momento t.

$X 5_{i t}=$ arrendamiento de la empresa i en el momento t.

$X 6_{i t}=$ tamaño de la empresa $i$ en el momento $t$.

3 Además de afectar el mercado de capitales, la crisis generada por Interbolsa afectó a la aerolínea Easyfly, debido a que Interbolsa a través de su fondo de capitalización adquirió el $40 \%$ de las acciones de la compañia por doce mil millones de pesos (Chacón, 2013). 
$X 7_{i t}=$ riesgo de la empresa $i$ en el momento t.

$X 8_{i t}=$ liquidez de la empresa $i$ en el momento $t$.

$d 1_{i t}=$ dummy estrategia de negocio.

$d 2_{i t}=$ dummy privado/público.

$d 3_{i t}=$ dummy ciclo económico.

$d 4_{i t}=$ dummy pertenece a un holding.

$\beta_{i}=$ coeficientes de regresión para cada una de las variables independientes.

$u_{i t}=$ término de error.

\section{RESULTADOS}

En la Tabla 1 se muestra la matriz de correlaciones de las variables regresoras, en donde a priori se observa que no hay evidencia de correlación fuerte entre las variables, a excepción del tamaño y el riesgo que tienen una correlación lineal positiva de 0.80 ; además, se observa que no existe relación lineal entre el arrendamiento y el crecimiento al tener una correlación de 0.007. Para analizar si existen problemas de multicolinealidad se acude al determinante de la matriz de correlaciones, el cual es de 0,07648 , es decir, que basado en el determinante no se observa una señal de multicolinealidad.

Tabla 1. Matriz de correlación

\begin{tabular}{lcccccccc}
\hline & Rentabilidad & Activos & Crecimiento & Combustible & Arrendamiento & Tamaño & Riesgo & Liquidez \\
\hline Rentabilidad & 1,000 & 0,450 & $-0,254$ & $-0,068$ & 0,083 & 0,223 & 0,175 & $-0,189$ \\
Activos & 0,450 & 1,000 & $-0,192$ & $-0,260$ & $-0,139$ & 0,040 & 0,239 & $-0,424$ \\
Crecimiento & $-0,254$ & $-0,192$ & 1,000 & 0,217 & $-0,007$ & $-0,211$ & $-0,118$ & 0,098 \\
Combustible & $-0,068$ & $-0,260$ & 0,217 & 1,000 & $-0,331$ & $-0,123$ & 0,031 & 0,404 \\
Arrendamiento & 0,083 & $-0,139$ & $-0,007$ & $-0,331$ & 1,000 & $-0,027$ & $-0,090$ & $-0,073$ \\
Tamaño & 0,223 & 0,040 & $-0,211$ & $-0,123$ & $-0,027$ & 1,000 & 0,809 & $-0,241$ \\
Riesgo & 0,175 & 0,239 & $-0,118$ & 0,031 & $-0,090$ & 0,809 & 1,000 & $-0,166$ \\
Liquidez & $-0,189$ & $-0,424$ & 0,098 & 0,404 & $-0,073$ & $-0,241$ & $-0,166$ & 1,000 \\
\hline Determinante & 0,07648 & & & & & & &
\end{tabular}

La ecuación se estimó mediante el método de mínimos cuadrados ordinarios, como se muestra en la Tabla 2. Se asumieron como supuestos que el crecimiento de Viva Colombia en el primer semestre de 2012 es cero, pues es el año en el cual la empresa comienza sus operaciones; para el riesgo se calculó la media móvil de tres periodos para no dejar constante esta variable; en el caso de las variables combustible y arrendamiento, se tomaron como referencia las cifras entregadas por la Aeronáutica Civil en su informe de costo operacional semestral, excluyendo a Satena y Avianca, las cuales publican sus estados financieros detallados. 
Tabla 2. Estimación con mínimos cuadrados ordinarios

Dependent Variable: NIVEL_DE_APALANCAMIENTO

Method: Panel Least Squares

Date: 06/02/19 Time: 16:43

Sample: 172

Periods included: 12

Cross-sections included: 6

Total panel (balanced) observations: 72

\begin{tabular}{ccccc}
\hline Variable & Coefficient & Std. Error & t-Statistic & Prob. \\
\hline ACTIVOS & 0.417260 & 0.100558 & 4.149465 & 0.0001 \\
ARRENDAMIENTO & 0.187728 & 0.597006 & 0.314449 & 0.7542 \\
COMBUSTIBLE & -0.312619 & 0.442715 & -0.706141 & 0.4827 \\
CRECIMIENTO & 0.018842 & 0.008566 & 2.199570 & 0.0315 \\
LIQUIDEZ & -0.101529 & 0.063479 & -1.599406 & 0.1147 \\
RENTABILIDAD & -0.530893 & 0.143076 & -3.710564 & 0.0004 \\
RIESGO & $-1.75 E-09$ & $4.44 E-10$ & -3.940781 & 0.0002 \\
TAMAÑO & 0.085256 & 0.027417 & 3.109585 & 0.0028 \\
C & -0.693428 & 0.617960 & -1.122124 & 0.2661 \\
\hline R-squared Rdjusted R-squared & 0.473158 & Mean dependent var & 0.895242 \\
S.E. of regression & 0.406258 & S.D. dependent var & 0.203816 \\
Sum squared resid & 0.157049 & Akaike info criterion & -0.748045 \\
Log likelihood & 1.553864 & Schwarz criterion & -0.463461 \\
F-statistic & 35.92961 & Hannan-Quinn criter. & -0.634751 \\
Prob (F-statistic) & 7.072569 & Durbin-Watson stat & 1.045872
\end{tabular}

Los resultados del $\mathrm{R}^{2}$ señalan que el nivel de apalancamiento es explicado en un $47 \%$ por las variables independientes. No obstante, al revisar la significancia individual de las variables, se determina que el arrendamiento, el combustible y la liquidez no son significativas dentro del modelo, ya que su $p$-valor es superior al $5 \%$. Al mismo tiempo, se realiza la estimación incluyendo una a una las variables dummy propuestas, las cuales no son significativas ${ }^{4}$. Por lo anterior, se eliminan del modelo las variables que no son estadísticamente significativas y se hace una nueva estimación.
Una vez se corre el modelo con las variables significativas, se procede a aplicar el test de Hausman para determinar si el método de efectos fijos o de efectos aleatorios es el más adecuado para estimar el modelo, el cual se puede observar en la Tabla 3. Como el p-valor es $<0.05$, se rechaza la hipótesis nula de igualdad con un $95 \%$ de confianza, lo que confirma que la estimación se debe llevar a cabo por el método de efectos fijos (Montero, 2005).

$4 \quad$ Para conocer las estimaciones, ver anexos. 
Tabla 3. Resultado Hausman Test

Correlated Random Effects - Hausman Test

Equation: Untitled

Test cross-section random

effects

\begin{tabular}{cccc}
\hline Test Summary & Chi-Sq. Statistic & Chi-Sq. d.f. & Prob. \\
\hline Cross-section random & 27.632730 & 5 & 0.0000 \\
\hline
\end{tabular}

En consecuencia, la variable riesgo dejó de ser significativa dentro del modelo, por lo que se eliminó y se obtuvo el modelo presentado en la Tabla 4. A este modelo se le practicaron pruebas de diagnóstico de residuales para validar los supuestos de media, normalidad (Jarque-Bera) y de no correlación de los errores (Breush-Pagan), cumpliendo con todos ellos 5 .

Tabla 4. Estimación método efectos fijos

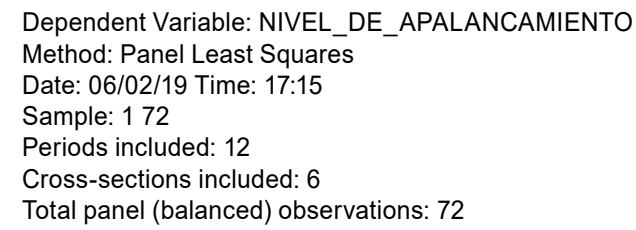

\begin{tabular}{lcccc}
\hline \multicolumn{1}{c}{ Variable } & Coefficient & Std. Error & t-Statistic & Prob. \\
\hline ACTIVOS & 0.680357 & 0.171927 & 3.957236 & 0.0002 \\
CRECIMIENTO & 0.019408 & 0.007914 & 2.452262 & 0.0170 \\
RENTABILIDAD & -0.487791 & 0.171664 & -2.841546 & 0.0061 \\
TAMAÑO & 0.096649 & 0.029904 & 3.231929 & 0.0020 \\
C & -1.248035 & 0.588289 & -2.121467 & 0.0379 \\
\hline
\end{tabular}

Effects Specification

\begin{tabular}{lclc}
\hline \multicolumn{1}{c}{ Cross-section fixed (dummy variables) } & & \\
\hline \multicolumn{1}{c}{ R-squared } & $\mathbf{0 . 6 1 0 4 0 0}$ & \multicolumn{1}{c}{ Mean dependent var } & $\mathbf{0 . 8 9 5 2 4 2}$ \\
\hline Adjusted R-squared & 0.553845 & S.D. dependent var & 0.203816 \\
S.E. of regression & 0.136138 & Akaike info criterion & -1.022046 \\
Sum squared resid & 1.149084 & Schwarz criterion & -0.705842 \\
Log likelihood & 46.79365 & Hannan-Quinn criter. & -0.896164 \\
F-statistic & 10.79306 & Durbin-Watson stat & 1.325888 \\
Prob (F-statistic) & 0.000000 & & \\
\hline
\end{tabular}

De este modelo se puede inferir que el nivel de apalancamiento es explicado en un $61.04 \%$ por la estructura de los activos, el crecimiento, la rentabilidad operacional y el tamaño de la compa- ñía. Parte de las variaciones del nivel de apalancamiento no puede ser explicado en un $38.96 \%$ por estas variables.

5 Para conocer las pruebas realizadas para validar los supuestos, ver anexos. 
Ahora bien, en cuanto a las variables explicativas, se puede decir que la estructura de activos tiene una relación positiva con el nivel de apalancamiento, esto es, que por cada $1 \%$ que aumente la estructura de activos, se espera que en promedio el nivel de apalancamiento se incremente en $0.68 \%$. Del mismo modo, el crecimiento tiene una relación positiva con el nivel de apalancamiento, es decir, que por cada $1 \%$ que aumente el crecimiento de la empresa, se espera que en promedio el nivel de apalancamiento se incremente en $0.02 \%$. De igual manera, el tamaño de la compañía tiene una relación positiva con el nivel de apalancamiento, esto es, que por cada $1 \%$ que aumente el tamaño, se espera que en promedio el nivel de apalancamiento se incremente en $0.09 \%$. Por el contrario, la rentabilidad operacional tiene una relación negativa con el nivel de apalancamiento, o sea, que por cada $1 \%$ que aumente la rentabilidad, se espera que en promedio el nivel de apalancamiento disminuya en $0.49 \%$.

\section{CONCLUSIONES}

La teoría de la estructura de capital en las últimas décadas ha sido objeto de varios estudios teóricos y empíricos debido a su relevancia en la toma de decisiones de la alta gerencia. Encontrar una relación deuda-capital óptima que satisfaga las necesidades particulares de financiamiento y a la vez garantice la sostenibilidad de la empresa, es mandatorio para todas las firmas. De ahí se origina la importancia de cono- cer cuáles son los determinantes de la estructura de capital que afectan esta relación en una industria $\mathrm{u}$ otra, de acuerdo con los postulados de las teorías modernas. Al revisar la literatura, para la industria área colombiana no se encontraron estudios empíricos sobre los determinantes de la estructura de capital que permitan conocer cuáles son los factores que inciden en el nivel de apalancamiento de las empresas, por tal razón, el presente estudio representa una primera aproximación, que contribuye al enriquecimiento de la literatura existente sobre estructura de capital.

Luego de una primera estimación mediante el método de MCO, el arrendamiento no es significativo, pues puede ser considerado como un sustituto de la deuda, tal como lo demuestra Erickson y Trevino (1994) en su investigación sobre los determinantes del arrendamiento, en el cual las empresas de acuerdo con el tipo de arrendamiento, ya sea financiero u operativo, pueden no afectar el nivel de endeudamiento de la compañía. De igual manera, el combustible no resulta ser un factor determinante en el nivel de apalancamiento, pero sí en sus utilidades. En cuanto al indicador de liquidez, si bien las empresas buscan mantenerlo en el tiempo, estadísticamente no tiene significancia al momento de determinar el nivel de apalancamiento de estas.

Se concluyó que el modelo se explica en mayor proporción por el método de efectos fijos, y se obtuvo como resultado que las variables rentabilidad operacio- 
nal, crecimiento, estructura de activos y tamaño de la compañía son los principales determinantes de la estructura de capital. La variable riesgo con este método dejó de ser significativa, por lo que fue excluida del modelo final al no tener efecto en el nivel de apalancamiento.

De acuerdo con el modelo estimado, se concluye que la variable rentabilidad tiene una relación negativa con el nivel de apalancamiento, es decir, que a medida que aumenta la rentabilidad disminuye el apalancamiento. Estos resultados concuerdan con lo que predice la teoría del Peking Order, pues en esta se asume que las empresas prefieren utilizar primero sus propios recursos antes de acudir al endeudamiento. Así lo demuestran los estudios empíricos de Tenjo et al. (2003), quienes validan esta relación. En cuanto a estudios en el sector aeronáutico, Khalil (2017) y Sewunet (2017) llegan a la misma conclusión.

Por otro lado, el crecimiento tiene una relación positiva con el nivel de apalancamiento, esto es, que a medida que aumenta el crecimiento, las empresas requerirán mayores recursos para completar sus planes de crecimiento, acudiendo primero al financiamiento interno hasta que este se agote y luego al externo antes de acudir al capital o a la emisión de acciones, tal como lo expresa la teoría del Peking Order. Estos resultados son consistentes con los obtenidos por Khalil (2017) y Alahyari (2014).
La estructura de activos también se constituye como un factor determinante y los resultados demuestran que existe una relación positiva entre el nivel de apalancamiento y la estructura de activos, pues los activos tangibles funcionan como garantía para contraer más deuda, en el caso en que la empresa tenga dificultades para honrar sus compromisos, protegiendo a los acreedores y reduciendo el riesgo de impago. Este resultado es respaldado por la teoría del Trade Off y por estudios realizados por Arango y Cadena (2018), Kasim y Nurhan (2018) y Sewunet (2017), en donde obtienen esta misma relación.

Así mismo, el tamaño de la compañía es otro determinante de la estructura de capital, el cual tiene una relación positiva con el nivel de apalancamiento, debido a que entre más grande sea la compañía mayor será su necesidad de apalancamiento, de acuerdo con el supuesto de que empresas de gran tamaño son más diversificadas que empresas de menor tamaño, lo que trae consigo una reducción en el riesgo de quiebra y un aumento en el poder de negociación con los acreedores. Así lo demuestra la teoría del Trade Off y los estudios de Khalil (2017) y Arango y Cadena (2018).

Estos resultados no logran establecer si la teoría del Pecking Order prevalece sobre la teoría del Trade Off o viceversa, porque mientras la rentabilidad y el crecimiento obtuvieron resultados sustentados por la teoría del Pecking Order, la estructura de activos y el tamaño de 
la compañía están respaldados por la teoría del Trade Off. Sin embargo, se logra establecer que los planteamientos de estas dos teorías logran explicar el nivel de apalancamiento que emplean las empresas analizadas en la industria aérea colombiana.

Finalmente es importante resaltar que esta investigación presenta resultados de acuerdo con el alcance planteado. Sin embargo, existe un amplio espacio de investigación en el sector aeronáutico que puede ser aprovechado por futuros investigadores. Si bien la presente investigación lleva a cabo una primera aproximación al estudio de los determinantes de la estructura de capital en la industria aérea colombiana, es recomendable ampliar el periodo de análisis para obtener resultados más precisos y que expliquen en mejor proporción la estructura de capital. Al mismo tiempo, es aconsejable incluir más aerolíneas de otras modalidades de transporte para observar el efecto general en la industria, a pesar de los limitantes que se puedan encontrar al momento de acceder a la información financiera de cada compañía. De igual manera, se recomienda incluir más variables explicativas que puedan tener influencia o afectar el nivel de apalancamiento de las empresas.

\section{DECLARACIÓN DE CONFLICTOS DE INTERÉS}

Los autores declaran que no existe conflicto de intereses. 


\section{REFERENCIAS}

Aeronáutica Civil de Colombia. (2017). La aviación en cifras. Aeronáutica Civil-Unidad Administrativa Especial.

AeronáuticaCivildeColombia.(2018a).Calidaddelserviciodetransporteaéreo.http:// www.aerocivil.gov.co/atencion/estadisticas-de-las-actividades-aeronauticas/ calidad-del-servicio-de-transporte-aereo

Aeronáutica Civil de Colombia. (2018b, abr.). Reglamentos aeronáuticos de Colombia. http://www.aerocivil.gov.co/normatividad/RAC/RAC\%20\%20 3\%20-\%20Actividades\%20A\%C3\%A9reas\%20Civiles.pdf

Aeronáutica Civil de Colombia.(2019a).Boletín costos deoperacion IIsemestre.http:// www.aerocivil.gov.co/atencion/estadisticas-de-las-actividades-aeronauticas/ boletines-financieros

Aeronáutica Civil de Colombia. (2019b, 17 ene.). 2018 cerró con un alto crecimiento de pasajeros movilizados vía aérea. http://www.aerocivil.gov.co/prensa/noticias/Pages/2018-cerr\%C3\%B3-con-un-alto-crecimiento-de-pasajeros-movilizados-v\%C3\%ADa-a\%C3\%A9rea.aspx

Aeronáutica Civil de Colombia. (2019c, 28 feb.). Más de 69 millones de viajeros se movilizaron. http://www.aerocivil.gov.co/prensa/noticias/Pages/M\%C3\%A1s-de-69-millones-de-viajeros-se-movilizaron-por-v\%C3\%ADa-a\%C3\%A9rea-en-2018.aspx

Akgül, E. F., \& Sigali, S. (Enero de 2018). Determinants of Capital Structure: An Application on BIST Transportation Index. https://www.researchgate.net/publication/322203097_Determinants_of_Capital_Structure_An_Application on_BIST_Transportation_Index

Alahyari, A. (2014, Aug.). Determinants of Profitability in the Airline Industry: A Comparison with Turkish Airlines. http://i-rep.emu.edu.tr:8080/xmlui/bitstream/handle/11129/1600/AlahyariAmirhassan.pdf?sequence=1

Arango, C. \& Cadena, C. (2018). La estructura de capital de las aerolíneas. https://repository.cesa.edu.co/bitstream/handle/10726/1863/MFC_812. pdf? sequence $=1$ \&isAllowed $=y$ 
Avianca Airlines. (2018, mayo). ¿Quiénes somos? https://www.avianca.com/ca/es/ sobre-nosotros/quienes-somos/

Avianca S.A. (2017). Estados financieros separados. Bogotá D.C.

Azofra, V. \& Fernández, A. I. (1999). Las finanzas empresariales 40 años después de las proposiciones de MM. Teorías y realidades. https://dialnet.unirioja.es/ servlet/articulo? codigo $=65346$

Banco Mundial. (2019a). Crecimiento del PIB (\% anual). https://datos.bancomundial. org/indicador/NY.GDP.MKTP.KD.ZG?end=2017\&locations $=$ CO\&start=2011

Banco Mundial. (2019b). Transporte aéreo, pasajeros transportados. https://datos. bancomundial.org/indicador/IS.AIR.PSGR

Baronio, A. \& Vianco, A. (2014, nov.). Datos de panel: guía para el uso de Eviews. http://www.econometricos.com.ar/wp-content/uploads/2012/11/datos-de-panel.pdf

Barcelonactiva. (2012, nov.). Estructuras low cost: modelo ganador. https://treball. barcelonactiva.cat/porta22/images/es/Barcelona_treball_capsula_sectorial_ transporte_aereo_noviembre2012_es_tcm24-22873.pdf

Briceño, D. C. \& López, O. (2011). Importancia del combustible en el transporte aéreo colombiano. https://repository.unimilitar.edu.co/bitstream/handle/10654/3217/ BricenoMorenoDianaCarolina2011.pdf?sequence $=2 \&$ isAllowed $=\mathrm{y}$

Capobianco, H. \& Fernandes, E. (2004, 12 mar.). Capital Structure in the World Airline Industry. Elsevier, (38), 421-434. https://afraassociation.org/wp-content/uploads/2017/01/Capital-structure-in-the-world-airline-industry.pdf

Chacón, J. (2013, 21 mayo). Interbolsa no manda en Easy Fly. https://www.elespectador.com/noticias/economia/interbolsa-no-manda-easy-fly-articulo-423360

CopaAirlines. (2017). Resultados trimestrales. https://copa.gcs-web.com/ financial-information/quarterly-results

Damodaran, A. (2019, 5 ene.). Capital Structure. Operating Lease Adjusted Values for Debt, Returns and Earnings (Global). http://pages.stern.nyu.edu/ adamodar/New_Home_Page/datacurrent.html 
Demirhan, A. (2009). Bankalarin sektörel paylarindaki değişimin nitel bağimli değişkenli panel veri modeliyle analizi. Ístanbul Üniversitesi Işsletme Fakültesi İşletme İktisadı Enstitüsü Dergisi-Yönetim, 20(64), 78-97. http://www.acarindex.com/dosyalar/makale/acarindex-1423905599.pdf

Díaz, O. (2015, 18 mayo). Análisis del desarrollo reciente del transporte aéreo en Colombia. Revista Transporte y Territorio, 122-143.

Durand, D. (1952). Costs of Debt and Equity Funds for Business: Trends and Problems of Measurement. En National Bureau of Economic Research. https:// www.nber.org/chapters/c4790.pdf

Easyfly. (2019). A cerca de nosotros. https://www.easyfly.com.co/terminos-de-uso/ acerca-de-nosotros

Erickson, S. \& Trevino, R. (1994). A Pecking Order Approach to Leasing: The Airline Industry Case. Journal of Financial and Strategic Decisions, 7(3), 71-81. https://pdfs.semanticscholar.org/fe5b/8a8ee48f748edc8326b8d412bc$60 \mathrm{f} 6 \mathrm{c} 82 \mathrm{ba} 5 . \mathrm{pdf}$

EViews. (2018, 1 nov.). EViews 9.5 Patch Installer. https://www.eviews.com/download/ev9download.html

Graham, B. \& Dodd, D. (1940). Security Analysis: The Classic 1940 Edition (2 ${ }^{\text {nd }}$ ed.). McGraw-Hill.

Guthmann, H., \& Dougall, H. (1955). Corporate Financial Policy (3rd ed.). Prentice-Hall.

International Credit Rating. (2018, ene.). Criterio: clasificación de compañias holdings y sus filiales. http://www.icrchile.cl/metodologias/Criterio-Clasificacionde-Companias-Holdings-y-sus-Filiales.pdf

Jensen, M., \& Meckling, W. (1976, oct.). Theory of the Firm: Managerial Behavior, Agency Costs and Ownership Structure. Journal of Financial Economics, 3(4), 305-360. https://www.sciencedirect.com/science/article/ pii/0304405X7690026X 
Kasim, K. \& Nurhan, A. (2018). Factors that Determine the Capital Structure: An Empirical Study on Low-cost Airlines. Scientific Annals of Economics and Business, 65(3), 227-246. https://www.researchgate.net/publication/327751039_ Factors_that_Determine_the_Capital_Structure_An_Empirical_Study_on_ Low-cost_Airlines

Khalil, F. M. (2017, nov.). Capital Structure in the Airline Industry. An Empirical Study of Determinants of Capital Structure. https://bspace.buid.ac.ae/bitstream/1234/1131/1/2014209061.pdf

Latam Airlines. (2017). Memoria 2017. http://memoria2017.marketinglatam.net/es/ nuestra-empresa/latam-cifras/1.php

LatamAirlines.(2019).Conócenos.https://www.latam.com/es_co/conocenos/historia/

Leggett, T. (2011, 19 nov.). BBC News. El auge de la aviación, ¿es sostenible? https:// www.bbc.com/mundo/noticias/2011/11/111118_economia_auge_aviacion_en

Modigliani, F. \& Miller, M. (1958, jun.). The Cost of Capital, Corporation Finance and the Theory of Investment. The American Economic Review, 48(3), 261-297. https:/gvpesquisa.fgv.br/sites/gvpesquisa.fgv.br/files/arquivos/terra_-_the cost_of_capital_corporation_finance.pdf

Montero, R. (2005, 22 sep.). Test de Hausman. https://www.ugr.es/ montero/matematicas/hausman.pdf

Morris, D. (2014, 14 ene.). Actualidad desregulación aérea. Revista TransPort. http://transport.ec/actualidad/desregulacion-aerea-el-triunfo-de-la-ideologia -sobre-la-evidencia/

Murray Z., F., \& Vidhan K., G. (2005, 10 feb.). Tradeoff and Pecking Order Theories of Debt. https://pdfs.semanticscholar.org/e05f/12544c3calaa47cf2c61ac4c56e4abeb7b03.pdf

Myers, S. (1977, nov.). Determinants of corporate borrowing. Journal of Financial Economics, 5(2), 147-175. Recuperado el 18 de Mayo de 2019, de https://www. sciencedirect.com/science/article/pii/0304405X77900150

Myers, S. (Julio de 1984). The Capital Structure Puzzle. The Journal of Finance, 39(3), 575-592. http://citeseerx.ist.psu.edu/viewdoc/download?doi $=10.1 \cdot 1.472 .3863 \&$ rep $=$ rep1\& type $=$ pdf 
Oficina de Regulación Económica Ministerio de Transporte. (2009). Revisión y análisis de la estructura de costos de transporte aéreo. Ministerio de Transporte. https://www.mintransporte.gov.co/descargar.php?idFile=4250

Ortiz, H. (2011). Análisis financiero aplicado. https://www.academia.edu/26760979/ Analisis_Financiero_Aplicado.pdf

Pearce, B. (2017, dic.). Economic Performance of the Airline industry - End2017 Update. https://www.iata.org/publications/economics/Reports/ Industry-Econ-Performance/Economic-Performance-of-the-Airlin e-Industry-end-year-2017-forecast-slides.pdf

Pedra, B. (2016, jul.). Compañias aéreas Low Cost, un estudio comparativo a través de la metodología de análisis de estudios contables. http://buleria.unileon.es/ bitstream/handle/10612/5528/76582626D_GF_julio16.pdf?sequence=1

Rivera, J. A. (2002, sep.). Teoría sobre la estructura de capital. http://www.scielo. org.co/scielo.php?script=sci_arttext\&pid=S0123-59232002000300002

Romero, A. (2019, 7 mayo). Colombia: un resumen histórico de nuestras crisis económicas y lo que nos espera. https://dernegocios.uexternado.edu.co/ controversia/colombia-un-resumen-historico-de-nuestras-crisis-economicas-y-lo-que-nos-espera/

Ross, S. (1977). La determinación de la estructura financiera: el enfoque de señalización de incentivos. RAND Corporation, 8(1), 23-40. https://www.jstor.org/ stable/3003485?seq=1\#page_scan_tab_contents

Satena. (2019). Quiénes Somos. https://www.satena.com/corporativo/naturaleza -de-satena/45

Schwartz, E. (1959, mar.). Theory of the Capital Structure of the Firm. The Journal of Finance, 14(1), 18-39. https://onlinelibrary.wiley.com/doi/abs/ 10.1111/j.15406261.1959.tb00483.x

Sewunet, A. (2017, feb.). Determinants of Capital structure of Airline industries. The Case of Major Airlines of Sub Saharan Africa. https://www.academia. edu/37238446/Determinants_of_Capital_structure_of_Airline_industries-The_case_of_major_airlines_of_Sub_Saharan_Africa 
Shyam, L., \& Myers, S. (1999). Testing Static Tradeoff Against Pecking Order Models of Capital Structure). Journal of Financial Economics, 51, 219-244. https://pdfs.semanticscholar.org/8ecf/61595b42ad97df818edecd79e1b1719e02a5.pdf

Smith, F. L., \& Cox, B. (2008). Econlib. http://www.econlib.org/library/Enc/ AirlineDeregulation.html

Tenjo, F., López, E., \& Zamudio, N. (2003, marzo). Determinantes de la estructura de capital de las empresas colombianas (1996-2002). Banco de la República. http://www.banrep.gov.co/sites/default/files/publicaciones/archivos/borra380. pdf

Titman, S., \& Wessels, R. (1988, marzo). The Determinants of Capital Structure Choice. The Journal of Finance, 43(1), 1-19. http://citeseerx.ist.psu.edu/ viewdoc/download?doi=10.1.1.462.6927\&rep=rep1\&type=pdf

Tomoiagă, I. C. (2014). The Effect of Capital Structure on Airline's Value. http:/ www.dafi.ase.ro/revista/9/Tomoiaga $\% 20$ Ionela $\% 20$ the $\% 20$ effect $\% 20$ of $\% 20$ capital\%20structure\%20on\%20airlines\%e2\%80\%99\%20value.pdf

VivaAir. (2018). Nuestra historia. https:/www.vivaair.com/co/viva-air/conocenos/ nuestra-historia

Zambrano, S. M. \& Acuña, G. A. (2011, dic.). Estructura de capital. Evolución teórica. Criterio Libre, 9(15), 81-102. https://dialnet.unirioja.es/descarga/articulo/3815888.pdf 


\section{Anexo 1. Estimaciones por MCO}

Tabla 1. Estimación por mco con variable dependiente ratio de apalancamiento

\begin{tabular}{|c|c|c|c|c|}
\hline $\begin{array}{l}\text { Dependent Variable: } \\
\text { Method: Panel Least } \\
\text { Date: } 06 / 04 / 19 \text { Time: } \\
\text { Sample: } 172 \\
\text { Periods included: } 12 \\
\text { Cross-sections includ } \\
\text { Total panel (balanced }\end{array}$ & $\begin{array}{l}\text { ATIO_DE_AP } \\
\text { quares } \\
1: 22\end{array}$ & ANCAMIE & & \\
\hline Variable & Coefficient & Std. Error & t-Statistic & Prob. \\
\hline ACTIVOS & -28.51845 & 38.63565 & -0.738138 & 0.4632 \\
\hline ARRENDAMIENTO & 108.5870 & 229.3780 & 0.473398 & 0.6376 \\
\hline COMBUSTIBLE & -196.2856 & 170.0972 & -1.153961 & 0.2529 \\
\hline CRECIMIENTO & -3.333715 & 3.291233 & -1.012908 & 0.3150 \\
\hline LIQUIDEZ & 9.771892 & 24.38969 & 0.400657 & 0.6900 \\
\hline RENTABILIDAD & 8.429035 & 54.97182 & 0.153334 & 0.8786 \\
\hline RIESGO & 1.97E-08 & $1.70 \mathrm{E}-07$ & 0.115489 & 0.9084 \\
\hline TAMAÑO & -3.505935 & 10.53408 & -0.332818 & 0.7404 \\
\hline $\mathrm{C}$ & 120.3243 & 237.4291 & 0.506780 & 0.6141 \\
\hline R-squared & 0.070263 & Mean de & dent var & 14.35946 \\
\hline Adjusted R-squared & -0.047798 & S.D. dep & dent var & 58.94818 \\
\hline S.E. of regression & 60.34056 & Akaike ir & criterion & 11.15435 \\
\hline Sum squared resid & 229381.9 & Schwar & riterion & 11.43894 \\
\hline Log likelihood & -392.5568 & Hannan- & inn criter. & 11.26765 \\
\hline F-statistic & 0.595140 & Durbin- & tson stat & 2.371425 \\
\hline Prob (F-statistic) & 0.778277 & & & \\
\hline
\end{tabular}

Tabla 2. Estimación por mco con variable dummy estrategia

\begin{tabular}{|c|c|c|c|c|}
\hline \multicolumn{5}{|c|}{$\begin{array}{l}\text { Dependent Variable: NIVEL_DE_APALA } \\
\text { Method: Panel Least Squares } \\
\text { Date: } 06 / 04 / 19 \text { Time: } 11: 29 \\
\text { Sample: } 172 \\
\text { Periods included: } 12 \\
\text { Cross-sections included: } 6 \\
\text { Total panel (balanced) observations: } 72\end{array}$} \\
\hline Variable & Coefficient & Std. Error & t-Statistic & Prob. \\
\hline ACTIVOS & 0.482377 & 0.085427 & 5.646643 & 0.0000 \\
\hline CRECIMIENTO & 0.022075 & 0.008974 & 2.459783 & 0.0166 \\
\hline RENTABILIDAD & -0.459990 & 0.166020 & -2.770693 & 0.0073 \\
\hline RIESGO & $-1.95 \mathrm{E}-09$ & $4.19 \mathrm{E}-10$ & -4.668118 & 0.0000 \\
\hline TAMAÑO & 0.089035 & 0.029522 & 3.015899 & 0.0037 \\
\hline ESTRATEGIA & -0.068269 & 0.059385 & -1.149594 & 0.2545 \\
\hline$C$ & -0.892488 & 0.587798 & -1.518358 & 0.1338 \\
\hline R-squared & 0.445742 & \multirow{2}{*}{\multicolumn{2}{|c|}{ Mean dependent var }} & \\
\hline Adjusted R-squared & 0.394580 & & & 0.895242 \\
\hline S.E. of regression & 0.158586 & \multicolumn{2}{|c|}{ S.D. dependent var } & 0.203816 \\
\hline Sum squared resid & 1.634726 & \multicolumn{2}{|c|}{ Akaike info criterion } & -0.752869 \\
\hline Log likelihood & 34.10330 & \multicolumn{2}{|c|}{ Schwarz criterion } & -0.531527 \\
\hline F-statistic & 8.712311 & \multicolumn{2}{|c|}{ Hannan-Quinn criter. } & -0.664752 \\
\hline Prob (F-statistic) & 0.000001 & \multicolumn{2}{|c|}{ Durbin-Watson stat } & 1.064944 \\
\hline
\end{tabular}


Tabla 3. Estimación por mco con variable dummy ciclo económico

Dependent Variable: NIVEL_DE_APALANCAMIENTO

Method: Panel Least Squares

Date: 06/04/19 Time: 11:32

Sample: 172

Periods included: 12

Cross-sections included: 6

Total panel (balanced) observations: 72

\begin{tabular}{ccccc}
\hline \multicolumn{1}{c}{ Variable } & Coefficient & Std. Error & t-Statistic & Prob. \\
\hline ACTIVOS & 0.507528 & 0.082824 & 6.127785 & 0.0000 \\
CRECIMIENTO & 0.018621 & 0.008618 & 2.160612 & 0.0344 \\
RENTABILIDAD & -0.572417 & 0.138362 & -4.137096 & 0.0001 \\
RIESGO & $-2.03 \mathrm{E}-09$ & $4.18 \mathrm{E}-10$ & -4.852387 & 0.0000 \\
TAMAÑO & 0.105903 & 0.025197 & 4.203052 & 0.0001 \\
CICLO & -0.017543 & 0.041197 & -0.425828 & 0.6716 \\
C & -1.245806 & 0.490557 & -2.539575 & 0.0135 \\
\hline R-squared & 0.436046 & Mean dependent var & 0.895242 \\
Adjusted R-squared & 0.383989 & S.D. dependent var & 0.203816 \\
S.E. of regression & 0.159967 & Akaike info criterion & -0.735527 \\
Sum squared resid & 1.663323 & Schwarz criterion & -0.514185 \\
Log likelihood & 33.47898 & Hannan-Quinn criter. & -0.647410 \\
F-statistic & 8.376271 & Durbin-Watson stat & 1.046602 \\
Prob (F-statistic) & 0.000001 & & \\
\hline
\end{tabular}

Tabla 4. Estimación por mco con variable dummy ciclo económico

Dependent Variable: NIVEL_DE_APALANCAMIENTO

Method: Panel Least Squares

Date: 06/04/19 Time: 11:39

Sample: 172

Periods included: 12

Cross-sections included: 6

Total panel (balanced) observations: 72

\begin{tabular}{ccccc}
\hline Variable & Coefficient & Std. Error & t-Statistic & Prob. \\
\hline ACTIVOS & 0.496783 & 0.097606 & 5.089671 & 0.0000 \\
CRECIMIENTO & 0.018091 & 0.009037 & 2.001950 & 0.0495 \\
RENTABILIDAD & -0.576871 & 0.141649 & -4.072529 & 0.0001 \\
RIESGO & $-2.03 E-09$ & $4.27 \mathrm{E}-10$ & -4.759863 & 0.0000 \\
TAMAÑO & 0.111684 & 0.028291 & 3.947736 & 0.0002 \\
HOLDING & -0.020377 & 0.073353 & -0.277797 & 0.7821 \\
C & -1.351554 & 0.528257 & -2.558516 & 0.0129 \\
\hline R-squared & 0.435143 & Mean dependent var & 0.895242 \\
Adjusted R-squared & 0.383003 & S.D. dependent var & 0.203816 \\
S.E. of regression & 0.160095 & Akaike info criterion & -0.733928 \\
Sum squared resid & 1.665985 & Schwarz criterion & -0.512586 \\
Log likelihood & 33.42141 & Hannan-Quinn criter. & -0.645811 \\
F-statistic & 8.345574 & Durbin-Watson stat & 1.061997 \\
Prob (F-statistic) & 0.000001 & & & \\
\hline
\end{tabular}


Tabla 5. Estimación por mco con variable dummy privado público

\begin{tabular}{|c|c|c|c|c|}
\hline \multicolumn{5}{|c|}{$\begin{array}{l}\text { Dependent Variable: NIVEL_DE_APALANCAMIENTO } \\
\text { Method: Panel Least Squares } \\
\text { Date: } 06 / 04 / 19 \text { Time: } 11: 41 \\
\text { Sample: } 172 \\
\text { Periods included: } 12 \\
\text { Cross-sections included: } 6 \\
\text { Total panel (balanced) observations: } 72\end{array}$} \\
\hline Variable & Coefficient & Std. Error & t-Statistic & Prob. \\
\hline $\begin{array}{l}\text { ACTIVOS } \\
\text { CRECIMIENTO } \\
\text { RENTABILIDAD } \\
\text { RIESGO } \\
\text { TAMAÑO } \\
\text { PRIVADO_PUBLICO } \\
\text { C }\end{array}$ & $\begin{array}{r}0.340120 \\
0.020305 \\
-0.394252 \\
-1.60 \mathrm{E}-09 \\
0.094322 \\
0.150588 \\
-1.017468\end{array}$ & $\begin{array}{l}0.120594 \\
0.008417 \\
0.162622 \\
4.68 \mathrm{E}-10 \\
0.025165 \\
0.079176 \\
0.488727\end{array}$ & $\begin{array}{c}2.820366 \\
2.412396 \\
-2.424341 \\
-3.427281 \\
3.748189 \\
1.901932 \\
-2.081876\end{array}$ & $\begin{array}{l}0.0064 \\
0.0187 \\
0.0181 \\
0.0011 \\
0.0004 \\
0.0616 \\
0.0413\end{array}$ \\
\hline $\begin{array}{l}\text { R-squared } \\
\text { Adjusted R-squared } \\
\text { S.E. of regression } \\
\text { Sum squared resid } \\
\text { Log likelihood } \\
\text { F-statistic } \\
\text { Prob (F-statistic) }\end{array}$ & $\begin{array}{l}0.464286 \\
0.414835 \\
0.155911 \\
1.580032 \\
35.32838 \\
9.388900 \\
0.000000\end{array}$ & $\begin{array}{l}\text { Mean depen } \\
\text { S.D. depende } \\
\text { Akaike info cr } \\
\text { Schwarz crite } \\
\text { Hannan-Quin } \\
\text { Durbin-Wats }\end{array}$ & $\begin{array}{l}\text { nt var } \\
\text { t var } \\
\text { erion } \\
\text { on } \\
\text { criter. } \\
\text { stat }\end{array}$ & $\begin{array}{c}0.895242 \\
0.203816 \\
-0.786900 \\
-0.565557 \\
-0.698782 \\
1.094539\end{array}$ \\
\hline
\end{tabular}

\section{Anexo 2. Pruebas de diagnóstico de residuales}

Tabla 6. Histogram - Normality Test

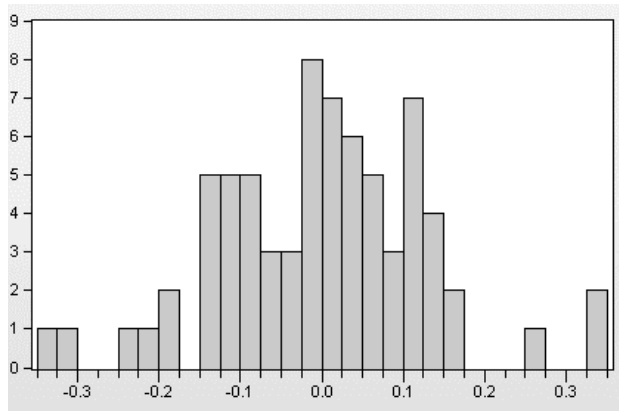

Series: Standardized Residuals
Sample 172
Pbservations 72

Tabla 7. Cross-Section Dependence Test

Residual Cross-Section Dependence Test

Null hypothesis: No cross-section dependence (correlation) in residuals

Equation: Untitled

Periods included: 12

Cross-sections included: 6

Total panel observations: 72

Cross-section effects were removed during estimation

\begin{tabular}{lccc}
\hline \multicolumn{1}{c}{ Test } & Statistic & d.f. & Prob. \\
\hline Breusch-Pagan LM & 19.54495 & 15 & 0.1901 \\
Pesaran scaled LM & -0.265655 & & 0.7905 \\
Bias-corrected scaled LM & -0.538382 & & 0.5903 \\
Pesaran CD & 0.968831 & & 0.3326
\end{tabular}

